\title{
Preliminary clinical results for PET/MR compared with PET/CT in patients with nasopharyngeal carcinoma
}

\author{
YONG CHENG $^{1 *}$, LE BAI $^{2 *}$, JINGJIE SHANG $^{1}$, YONGJIN TANG $^{1}$, XUEYING LING $^{1}$,

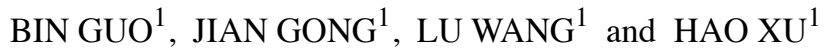 \\ Departments of ${ }^{1}$ Nuclear Medicine and ${ }^{2}$ Radiology, The First Affiliated Hospital of Jinan University, \\ Guangzhou, Guangdong 510630, P.R. China
}

Received April 17, 2019; Accepted September 9, 2019

DOI: $10.3892 /$ or.2019.7392

\begin{abstract}
The present study aimed to assess the performance of positron emission tomography-magnetic resonance imaging (PET/MR) for the visualization and characterization of lesions. In addition, the present study investigated whether the apparent diffusion coefficient (ADC) and intravoxel incoherent motion parameters exhibited any significant correlation with standardized uptake values (SUV) in patients with nasopharyngeal carcinoma (NPC). A total of 35 patients with NPC underwent whole body PET-computed tomography (CT) and head and neck MR imaging (MRI) scans using the PET/CT-MRI system. Image quality, lesion conspicuity and the diagnostic confidence of PET/CT, T1 weighted (T1w) PET/MR and T2w PET/MR imaging were assessed. The true diffusion coefficient (D), the pseudo-diffusion coefficient or diffusion within the microcirculation $\left(\mathrm{D}^{*}\right)$, and the perfusion fraction or the contribution of water moving in the capillaries (f), and ADC, were calculated. The correlation between the ADC, D*, $\mathrm{D}$ and $\mathrm{f}$ values and the SUV were analyzed using Pearson's correlation analysis. Similar image quality was obtained using PET/CT, T1w PET/MR and T2w PET/MR imaging. However, the T1w PET/MR and T2w PET/MR imaging were more effective than PET/CT in analyzing the lesion conspicuity of the primary tumors and lymph nodes. In addition, T2w PET/MR imaging was more efficient than T1w PET/MR imaging in analyzing primary tumors and lymph nodes. Pearson's correlation analysis showed no significant correlation between the SUV and ADC, and D*, D and f values in NPC. The present results suggested that the application of PET/MR is feasible and could serve as a reliable alternative to PET/CT, while SUV
\end{abstract}

Correspondence to: Professor Hao Xu, Departments of Nuclear Medicine, The First Affiliated Hospital of Jinan University, 613 West Huangpu Road, Guangzhou, Guangdong 510630, P.R. China E-mail:txh@jnu.edu.cn

${ }^{*}$ Contributed equally

Key words: positron emission tomography-magnetic resonance imaging, nasopharyngeal carcinoma, standardized uptake value, diffusion weighted imaging, intravoxel incoherent motion and $\mathrm{ADC}, \mathrm{D}^{*}, \mathrm{D}$ and $\mathrm{f}$ values were identified as independent biomarkers in NPC.

\section{Introduction}

Nasopharyngeal carcinoma (NPC) is a cancer that is distinct from other epithelial tumors of the head and neck region (1). NPC has a peculiar geographical distribution, and its incidence is higher in Southern China and South-Central Asia, representing the most frequent carcinoma of the head and neck region in these geographical regions $(1,2)$. Efficient imaging techniques are crucial for staging and planning the correct radiotherapy treatment for patients with NPC (1). Integrated positron emission tomography-computed tomography (PET/CT) using fluorine-18 fludeoxyglucose $\left({ }^{18} \mathrm{~F}-\mathrm{FDG}\right)$, which provides metabolic parameters such as the standardized uptake value (SUV) and total lesion glycolysis, is a widely used techniques in cancer staging assessment and for predicting clinical outcomes $(3,4)$. $\mathrm{PET} / \mathrm{CT}$ is sensitive and accurate for the detection of nodal metastases and distant metastases, but lacks the soft tissue resolution compared to the magnetic resonance imaging (MRI) when analyzing primary tumor (5-7). Similarly, MRI provides a higher resolution than $\mathrm{CT}$ in terms of assessing parapharyngeal spaces, marrow infiltration of the skull base, intracranial disease and deep cervical nodes in NPC (1). Additionally, the development of the MRI technology, including diffusion and perfusion MRI, has added the potential to analyze biological features in addition to the structural analysis (1).

PET and MR both provide functional parameters: Cellular glucose metabolism as expressed by SUV can be identified by PET, and the random Brownian motion of water molecules expressed by the apparent diffusion coefficient (ADC) can be analyzed by MRI (8). Many malignant tumors show a significantly increased glucose uptake, and malignant tumor with high cellularity exhibit higher diffusions restrictions, which are indicated by a low ADC (9-14). Therefore, based on the hypothesis that SUV and ADC provide similar information about tumors and show a negative correlation, many previous studies reported the correlation between SUV and ADC for various tumors, but the results are not homogenous (8-19). An inverse correlation was demonstrated in non-small cell lung cancer (9), breast cancer (10-12), gastrointestinal stromal tumor (13) and cervical cancer (14). However, data from 
patients with lymphoma suggested that SUV and ADC are independent biomarkers $(15,16)$. For head and neck squamous cell carcinoma (HNSCC), it is unclear whether there is a significant correlation between SUV and ADC $(8,17-19)$.

The movement of water molecules contributing to the signal in diffusion-weighted imaging (DWI) are caused by different factors: Extracellular space diffusion, intracellular space diffusion and intravascular space diffusion (or perfusion) (20-22). Accordingly, the signal attenuation on conventional mono-exponential DWI does not always present a linear relationship, and it is difficult to calculate an accurate ADC value (23). In order to separate the motion of water molecules due to perfusion in the microcirculation from that due to diffusion in the extravascular space, the intravoxel incoherent motion (IVIM) imaging method, first described by Le et al (22) may be used. IVIM is a powerful imaging technique that uses DWI with multiple $b$ values followed by bi-exponential curve fitting. IVIM can provide the true diffusion coefficient (D), the pseudo-diffusion coefficient or diffusion within the microcirculation $\left(\mathrm{D}^{*}\right)$, and the perfusion fraction or the contribution of water moving in the capillaries (f). Several studies have reported the application of IVIM in NPC, and these previous reports have shown that IVIM is a feasible technique $(24,25)$. In addition, IVIM parameters have been associated with clinical stage (26) and dynamic contrast enhanced-MRI parameters (25). IVIM can be applied to differentiating lymphoma (27) and enlarged adenoids (24), and can predict the early treatment response of patients with NPC $(28,29)$.

PET/MR, a new medical imaging tool, combines the metabolic information of PET with the anatomical detail of MR, thus exhibiting good potential for clinical application (30). There are two types of PET/MR systems: Sequential PET/MR, in which PET and MR data are acquired sequentially, either in a single room or using a tri-modality PET/CT-MRI system, and simultaneous PET/MR, in which PET and MR are integrated systems $(30,31)$. Several previous studies have investigated the diagnostic performance of PET/MR compared with $\mathrm{PET} / \mathrm{CT}$ in head and neck cancer (32-38). Certain previous studies examined the clinical potential of PET/MR in NPC using simultaneous PET/MR, and no significant correlation between SUV and ADC in NPC was found by conventional mono-exponential DWI $(39,40)$. However, the feasibility of a routine application of PET/CT-MRI and the correlation between the IVIM parameters and SUV need to be further explored in NPC. Therefore, the aim of the present study was to assess the performance of PET/MR for the visualization and characterization of lesions and to further explore the correlation between SUV and ADC value, between SUV and D value, between SUV and $\mathrm{D}^{*}$ value, and between SUV and $\mathrm{f}$ value using sequential PET/MR in NPC.

\section{Materials and methods}

Patients. The present study was approved by the Ethics Committee of The First Affiliated Hospital of Jinan University, and written informed consent was obtained from each participant. Inclusion criteria were as follows: All patients were diagnosed with NPC using histology, all underwent whole body ${ }^{18} \mathrm{~F}-\mathrm{FDG} \mathrm{PET} / \mathrm{CT}$, and all patients were examined with regular head and neck using a PET/CT-MRI system, and none received anti-tumor treatment before the PET/CT-MRI scan. Exclusion criteria were the following: Patients under 18 years of age, patients with claustrophobia and patients with MRI-incompatible medical devices, including cardiac pacemakers, cochlear implants and insulin pumps.

Between June 2015 and October 2017, 35 patients (7 women and $28 \mathrm{men}$; age, $50.4 \pm 11.7$ years; age range, $24-77$ years) were included. All patients presented non-keratinizing undifferentiated carcinoma. There were two patients at stage T1, four at T2, 14 at T3 and 15 at T4 primary tumors (41). Nodal metastases and distant metastases were found in eight and 31 patients, respectively. All of the patients underwent additional multiple b-value DWI scans. However, two patients were excluded from further evaluation of the correlation between the SUV and $\mathrm{ADC}$, and $\mathrm{D}^{*}, \mathrm{D}$ and $\mathrm{f}$ values, because of image distortions in the DW images.

Imaging modalities. All of the patients underwent a tri-modality PET/CT-MRI (full ring; time-of-flight Discovery PET/CT 690; 3T Discovery MR 750; GE Healthcare) comprising a sequential whole-body ${ }^{18} \mathrm{~F}$-FDG PET/CT and a regular head and neck MRI. The patients were positioned on a dedicated shuttle board, facilitating their transport from the PET/CT to the MRI system without movement.

PET/CT imaging. The patients fasted for at $>6 \mathrm{~h}$ before scanning and their blood glucose levels were measured immediately before the tracer injection. The patients were intravenously administered with ${ }^{18} \mathrm{~F}-\mathrm{FDG}$ with an activity range of $0.08-0.10 \mathrm{mCi} / \mathrm{kg}$ body weight, and the data acquisition was performed after an uptake time of 50-70 min. The data acquisition was performed from the skull base to the mid-thigh, which were used for both the PET and CT modalities. The $\mathrm{CT}$ data were acquired with an automatic dose modulation at $120 \mathrm{kV}$, a range of $80-160 \mathrm{~mA}$, a $512 \times 512$ image matrix, a field of view (FOV) of $50 \mathrm{~cm}$, a noise index of 30 , reconstructed slice thickness of $3.75 \mathrm{~mm}$, and a slice interval of $3.27 \mathrm{~mm}$. The PET protocol encompassed seven bed positions with a scan duration of $120 \mathrm{sec}$ per bed position. The PET data was acquired in 3D time-of-flight mode using the adaptive statistical iterative reconstruction (42) with an axial FOV of $70 \mathrm{~cm}$, reconstructed slice thickness of $3.27 \mathrm{~mm}$, a slice interval of $3.75 \mathrm{~mm}$ and a $192 \times 192$ image matrix. The attenuation correction was based on the whole-body CT.

MRI. After PET/CT procedure, the patients were transferred to the MRI via a dedicated shuttle board. The MRI scans were acquired using the dedicated RF-covered nasopharynx coil. No contrast medium was applied during MRI. The imaging acquisition parameters for the applied MR sequences are presented in Table I.

Image analysis. All the images were analyzed by two investigators who evaluated both data sets. One investigator was an experienced nuclear medicine physician with 4 years of experience in MRI, and the second was a radiologist with additional experience in PET/CT. The PET, CT, and MRI data were sent to a dedicated review workstation (GE Advantage workstation, version 4.6; GE Healthcare Life Sciences) that 
Table I. Acquisition parameters for the applied MR sequences.

\begin{tabular}{|c|c|c|c|c|c|}
\hline \multirow[b]{2}{*}{ Parameter } & \multicolumn{2}{|c|}{ FSE T1WI } & \multicolumn{2}{|c|}{ FRFSE T2WI } & \multirow{2}{*}{$\frac{\text { SS-EPI DWI }}{\text { Axial }}$} \\
\hline & Axial & Sagittal & Axial & Coronal & \\
\hline TR/TE, ms & $466 / 14.1$ & $350 / 13.8$ & $3,877 / 81.3$ & $3,023 / 108.2$ & $3,000 / 80$ \\
\hline $\mathrm{FOV}, \mathrm{cm}$ & $20 \times 20$ & $26 \times 26$ & $20 \times 20$ & $26 \times 26$ & $24 \times 24$ \\
\hline Thickness, mm & 3.5 & 4.0 & 3.5 & 4.0 & 3.5 \\
\hline Slice space, $\mathrm{mm}$ & 0.5 & 1.5 & 0.5 & 0.5 & 0.5 \\
\hline Matrix & $288 \times 224$ & $320 \times 256$ & $288 \times 256$ & $288 \times 256$ & $128 \times 128$ \\
\hline Acceleration factor & 2 & 2 & 2 & 2 & 2 \\
\hline Bandwidth, kHz & 31.2 & 31.2 & 41.7 & 41.7 & 250 \\
\hline Flip angle, ${ }^{\circ}$ & 111 & 111 & 111 & 111 & 90 \\
\hline B Values, $\mathrm{s} / \mathrm{mm}^{2}$ & NA & NA & NA & NA & $\begin{array}{c}0,50,200,500,800,1,000 \\
1,500,2,000,3,000\end{array}$ \\
\hline NEX & 2 & 1 & 3 & 3 & $1,1,1,1,2,2,4,4,6$ \\
\hline
\end{tabular}

FSE T1WI, fast spin-echo T1-weighted imaging; FRFSE T2WI, fast recovery fast spin-echo T2-weighted imaging; SS-EPI DWI, single-shot echo planar diffusion-weighted imaging; TR, repetition time; TE, echo time; FOV, field of view; NEX, number of excitations; NA, not applicable.

allowed the simultaneous review of PET, CT and MR images side by side or in fused/overlay mode (PET/CT, T1w PET/MR and T2w PET/MR imaging).

Visualization and characterization of lesions. Each PET examination was evaluated for the presence of PET-positive lesions within the body area that was covered by the head and neck MR imaging. NPC cases were considered PET-positive primary tumors if their maximum standardized uptake value (SUVmax) was markedly higher than the surrounding background activity. Cervical lymph node metastases were defined based on both functional and morphological criteria. The functional criteria used for the PET compound were: i) Visually detectable metabolic activity higher than normality; or ii) asymmetric metabolic activity greater than that of normal-appearing lymph nodes at the same level in the contralateral neck (43). The morphological criteria for lymph node metastases on CT and MRI included (44): i) A shortest axial diameter of $>5 \mathrm{~mm}$ in the retropharyngeal region and $>10 \mathrm{~mm}$ in other regions of the neck; ii) any nodes with necrosis or extracapsular spread; and iii) a group with $>2$ nodes with a shortest axial diameter of $\sim 5 \mathrm{~mm}$ in the retropharyngeal region and $\sim 10 \mathrm{~mm}$ in other regions of the neck.

Image quality. A 3-point scale was used for the assessment of image quality: i) $1=$ substantial artefacts with insufficient image quality for further assessment; ii) 2=mild artefacts with sufficient image quality for lesion assessment; and iii) $3=$ absence of relevant artefacts.

Lesion conspicuity. Lesion conspicuity was graded on a 4-point scale according to the lesion delineation: i) 1, not detectable or poorly delineated (less than $25 \%$ of lesion borders definable); ii) 2 , moderately delineated lesion borders (25-50\% of borders definable); iii) 3 , well delineated lesion borders $(50-75 \%$ of borders definable); and iv) 4 , excellently delineated ( $>75 \%$ of lesion borders definable).
Diagnostic confidence. To identify whether CT imaging and MR imaging can increase the diagnostic confidence for PET-positive primary tumors when characterizing tumor lesions, three scores were assigned: A grade of 1 indicated that MR imaging provided more relevant information than CT imaging, including infiltration of the adjacent structures such as the skull-base bone and prevertebral muscle. A grade of -1 indicated that $\mathrm{CT}$ imaging provided more information than MR imaging, including subtle bone destruction not visible on MR imaging. A grade of 0 indicated that both CT imaging and MR imaging provided the same information.

$S U V, A D C$, and $D^{*}, D$ and $f$ values measurements. Using the dedicated review workstation (GE Advantage workstation; version 4.6; GE Healthcare Life Sciences), SUV of each voxel was calculated according to the following equation:

$$
\mathrm{SUV}=\frac{\text { measured radioactivity concentration }[\mathrm{Bq} / \mathrm{mL}]}{\text { injected radioactivity }[\mathrm{Bq}] /(\text { lean body mass }[\mathrm{kg}] \mathrm{x} 1000)}
$$

The SUVmax and mean SUV (SUVmean) were calculated on the basis of activity values in regions of interest manually placed on the area of the tumor containing the highest-SUV pixel. The margin of the tumor was determined by visual inspection.

Subsequently, each investigator measured the IVIM parameters ( $D^{*}, \mathrm{D}$ and $\mathrm{f}$ values) using the standard software on the workstation. The bi-exponential model from an IVIM sequence was expressed using the following equation, as described by Le et al (45): $\mathrm{Sb} / \mathrm{S} 0=(1-\mathrm{f}) \exp (-\mathrm{b} \mathrm{D})+\mathrm{f} \exp$ $\left[-b\left(D^{*}+D\right)\right]$. Where $\mathrm{Sb}$ is the signal intensity with diffusion gradient $b ; S 0$ is the signal intensity for $a b$ value of 0 $\mathrm{s} / \mathrm{mm}^{2} ; \mathrm{D}$ is the true diffusion coefficient as reflected by pure molecular diffusion; $f$ is the fractional perfusion related to microcirculation; and $\mathrm{D}^{*}$ is the pseudo-diffusion coefficient related to perfusion.

In addition, each investigator extracted the DW image with $b$ values of 0 and $1,000 \mathrm{~s} / \mathrm{mm}^{2}$ to calculate the ADC 
A

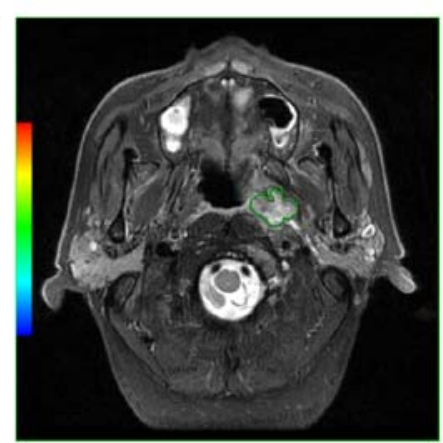

D

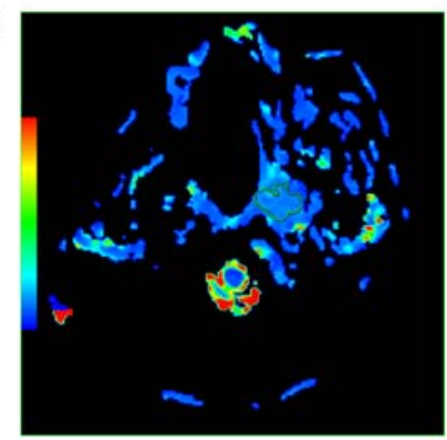

B

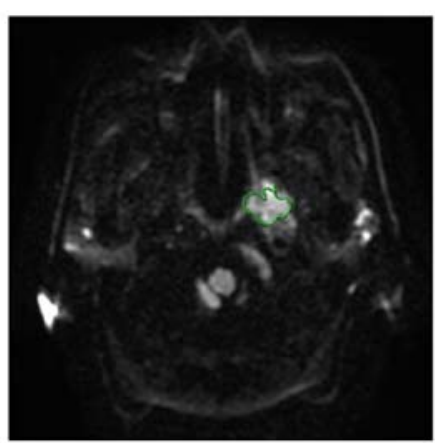

E

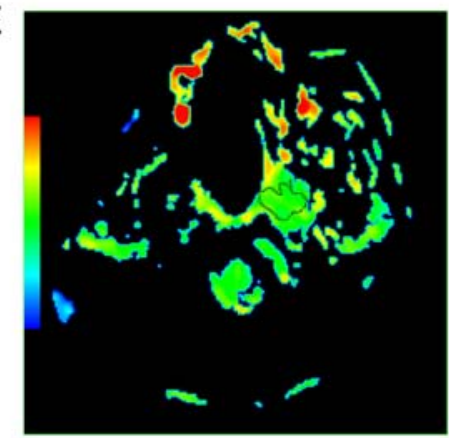

C

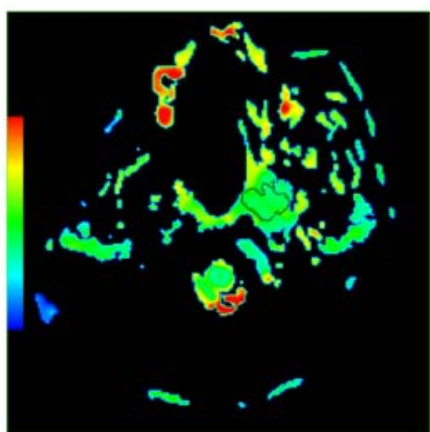

F

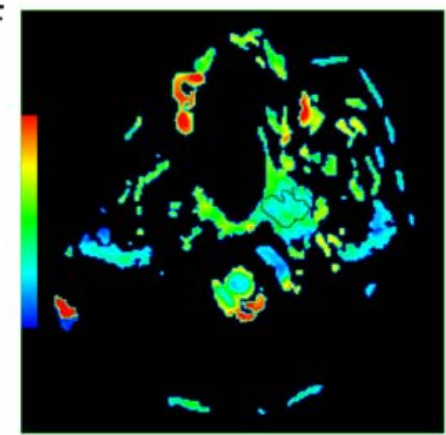

Figure 1. A 62-year-old male patient with NPC. (A) Axial T2w imaging and (B) axial DW imaging showing a region of interest around the margin of NPC. (C) ADC, (D) D*, (E) D and (F) f maps. The ADC, D*, D, and f values were $8.43 \times 10^{-4} \mathrm{~mm}^{2} / \mathrm{s}, 4.27 \times 10^{-3} \mathrm{~mm}^{2} / \mathrm{s}, 5.59 \times 10^{-4} \mathrm{~mm}^{2} / \mathrm{s}$, and 0.291 , respectively. NPC, nasopharyngeal carcinoma; ADC, apparent diffusion coefficient; D, true diffusion coefficient; D*, pseudo-diffusion coefficient.

A

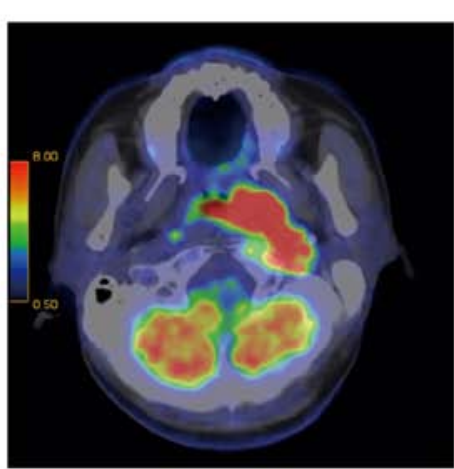

D

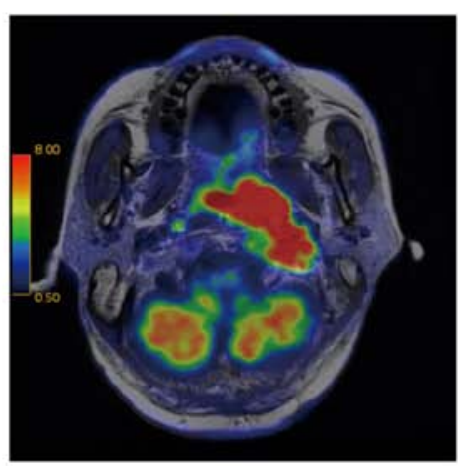

B

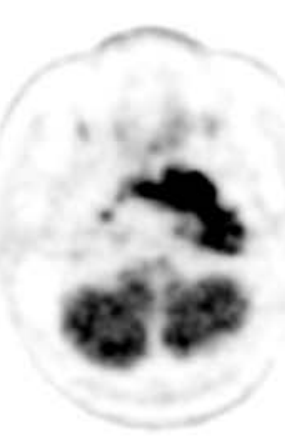

E

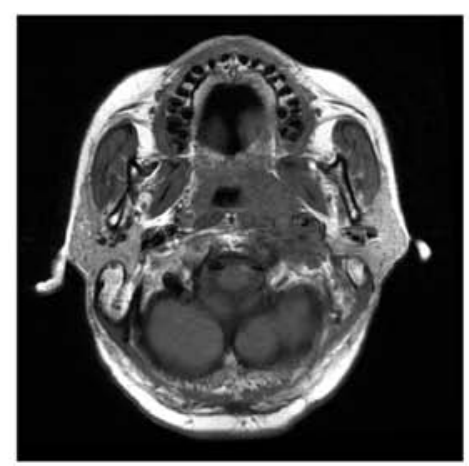

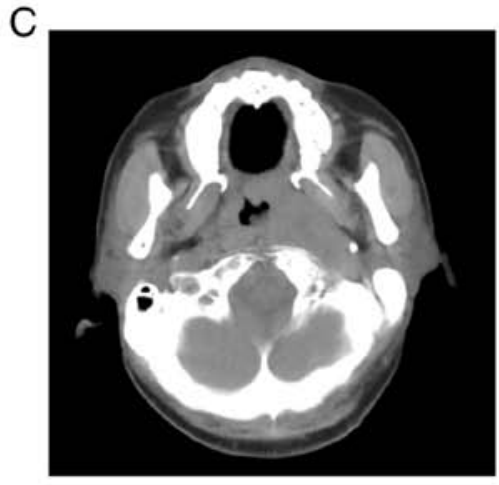

$\mathrm{F}$

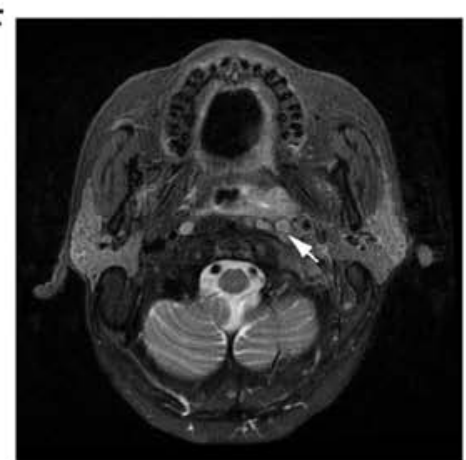

Figure 2. A 58-year-old male patient with NPC. The left retropharyngeal node was merged with the primary tumor. (A) PET/CT imaging. (B) PET and (C) CT did not separate retropharyngeal nodes from the adjacent primary tumor. (D) T1w PET/MR imaging. (E) T1w imaging. (F) T2w imaging was able to separate the left retropharyngeal node from the primary tumor in the adjacent posterolateral nasopharynx (indicated by an arrow). PET, positron emission tomography; MR, magnetic resonance imaging; CT, computed tomography; T1w, T1 weighted; NPC, nasopharyngeal carcinoma.

using a mono-exponential model with the following equation: $\mathrm{Sb} / \mathrm{S} 0=\exp (-b \mathrm{ADC})$.
The regions of interest were manually drawn to delineate the borders of the primary tumor, in order to cover as much of 
the tumor as possible on each slice of the axial DW images that corresponded to the high-signal area on T2-weighted imaging with fat suppression, and avoiding apparent cystic changes, necrosis and adjacent anatomic structures (Fig. 1). The IVIM parameters obtained for each tumor were calculated on a pixel-by-pixel basis and expressed as the mean values of all pixels within the volume of interest. The minimum ADC (ADCmin) and mean ADC (ADCmean) were calculated for all primary tumors, with the ADCmin defined as the lowest ADC value within the whole tumor on the ADC map. Therefore, for each primary tumor, the ADCmean was defined as the mean value of all the tumor pixels on the ADC map.

Statistical analysis. The data are presented as the mean \pm SD. The differences between image quality and lesion conspicuity in PET/CT, T1w PET/MR, and T2w PET/MR imaging were analyzed using Friedman $M$ test. The Nemenyi post hoc test was used to further evaluate the differences between two methods. The inter-observer agreement for the ADC, D*, D and $f$ values was evaluated using intraclass correlation coefficients (ICC). Pearson's correlation coefficient analysis was used to evaluate the correlation between the ADC (ADCmin and ADCmean), D*, D and $f$ values and the SUV (SUVmax and SUVmean). $\mathrm{P}<0.05$ was considered to indicate a statistically significant difference.

\section{Results}

Lesion detection. A total of 99 PET-positive lesions were evaluated, including 35 primary tumors and 64 lymph nodes. Among the 64 lymph nodes, there were 25 in the retropharyngeal space and 39 in other regions of the neck. All of the lesions had increased ${ }^{18} \mathrm{~F}$-FDG uptake and all of the primary tumors could be detected by PET/CT, T1w PET/MR, and T2w PET/MR imaging. For the lymph nodes, PET/CT and T1w PET/MR imaging detected 61 lymph nodes, and the three other retropharyngeal lymph nodes were not detected by either PET/CT or T1w PET/MR imaging. However, T2w PET/MR imaging detected all of the lymph nodes. T2w PET/MR imaging is presented in Fig. 2.

Image quality. No statistically significant differences were found between the artefact grading, an indicator of image quality, in T1w PET/MR, T2w PET/MR imaging and PET/CT (Tables II and III). The artefacts did not originate in the nasopharynx but in the oral cavity, and these artefacts were caused by dental implants and, in one case, by eyeball motion. All of the dental implant artefacts were found in T1w PET/MR, T2w $\mathrm{PET} / \mathrm{MR}$ and PET/CT, whereas the eyeball motion artefacts were found only in T2w PET/MR imaging (Table II).

Correlations between the $S U V$ and $A D C$, and $D, D^{*}$ and $f$ values. There was no correlation between the ADC (ADCmin and ADCmean), D, D* and f values and the SUV (SUVmax and SUVmean) for the primary tumors (Table IV; Figs. 3 and 4).

Lesion conspicuity. Regarding the conspicuity of the primary tumors and lymph nodes, there were significant differences between PET/CT, T1w PET/MR imaging and T2w PET/MR imaging (Tables III and V). Further comparison indicated
Table II. Artefact graded for the three techniques.

\begin{tabular}{lccc}
\hline Grade & $\begin{array}{c}\text { PET/CT } \\
(\%)\end{array}$ & $\begin{array}{c}\text { T1w PET/MR } \\
(\%)\end{array}$ & $\begin{array}{c}\text { T2w PET/MR } \\
(\%)\end{array}$ \\
\hline No & 88.6 & 88.6 & 80.0 \\
Mild & 8.6 & 11.4 & 20.0 \\
Substantial & 2.8 & 0 & 0
\end{tabular}

PET, positron emission tomography; MR, magnetic resonance imaging; CT, computed tomography; T1w, T1 weighted.

that T1w PET/MR imaging and T2w PET/MR imaging were significantly more efficient than PET/CT in primary tumors (both $\mathrm{P}<0.01$ ) and in lymph nodes (both $\mathrm{P}<0.01$; Fig. 5). T2w $\mathrm{PET} / \mathrm{MR}$ imaging was more efficient than T1w PET/MR imaging in primary tumors and in lymph nodes (data not shown).

Diagnostic confidence. Regarding primary tumors characterization, T1w PET/MR imaging was considered superior to $\mathrm{PET} / \mathrm{CT}$ in $71.4 \%$ of cases, equal to PET/CT in $22.9 \%$ of cases, and inferior to PET/CT in $5.7 \%$ of cases. T2w PET/MR imaging was considered superior to PET/CT in $77.2 \%$ of cases (Fig. 6), equal to PET/CT in $17.1 \%$ of cases, and inferior to $\mathrm{PET} / \mathrm{CT}$ in $5.7 \%$ of cases.

SUV and $A D C$, and $D^{*}, D$ and $f$ values. The mean SUVmax and mean SUVmean values of all primary tumors were 12.6 \pm 4.9 (range 4.7-25.9) and 7.6 \pm 3.0 (range 2.6-16.5), respectively (data not shown). The means of the ADCmin, ADCmean, D*, D and $\mathrm{f}$ values of all primary tumors were $(7.78 \pm 1.25) \times 10^{-4} \mathrm{~mm}^{2} / \mathrm{s}$, $(8.14 \pm 1.23) \times 10^{-4} \mathrm{~mm}^{2} / \mathrm{s},(5.44 \pm 2.01) \times 10^{-3} \mathrm{~mm}^{2} / \mathrm{s},(5.13 \pm 0.59)$ $\mathrm{x} 10^{-4} \mathrm{~mm}^{2} / \mathrm{s}$ and $28.68 \pm 6.40 \%$, respectively.

Inter-observer agreement. The ICCs (95\% CI) of the inter-observer reproducibility for the measurements of the ADC, D*, D and f values were 0.990 (0.983 to 0.994), 0.970 (0.940 to 0.985$), 0.898$ (0.801 to 0.948$)$ and 0.969 (0.938 to 0.985), respectively, which suggested good inter-observer reproducibility and consistency.

\section{Discussion}

PET/CT has high sensitivity in diagnosing NPC, and MR shows a sensitivity of $\sim 100 \%$ (46). In the present study, a total of 35 NPC primary tumors had increased ${ }^{18} \mathrm{~F}$-FDG uptake and were detected on PET/CT and PET/MR, in line with a previous study (39). However, Chen et al (47) reported only 15 patients with FDG uptake out of 20 newly-diagnosed patients with NPC. This difference may be due to the pathological subtype of NPC investigated in the present study. In fact, the NPC examined was a non-keratinizing undifferentiated carcinoma of a relatively simple type, which resulted in high ${ }^{18} \mathrm{~F}-\mathrm{FDG}$ uptake from the NPC. In terms of tumor conspicuity, PET/CT did not efficiently delineated the tumor borders, whereas $85.7 \%$ of lesion borders could be efficiently delineated by $\mathrm{T} 2 \mathrm{~W}$ $\mathrm{PET} / \mathrm{MR}$ imaging. These present results showed that tumor 
Table III. Image quality and lesion conspicuity in the three imaging techniques.

\begin{tabular}{lcccc}
\hline Parameter examined & PET/CT & T1w PET/MR & T2w PET/MR & P-value \\
\hline $\begin{array}{l}\text { Image quality, median (range) } \\
\text { Lesion conspicuity }\end{array}$ & $3(1-3)$ & $3(2-3)$ & $3(2-3)$ & 0.174 \\
Primary tumors, median (range) & $2(1-3)$ & $3(2-4)$ & $4(3-4)$ & $<0.001$ \\
Lymph nodes, median (range) & $3(1-4)$ & $4(1-4)$ & $4(3-4)$ & $<0.01$ \\
\hline
\end{tabular}

PET, positron emission tomography; MR, magnetic resonance imaging; CT, computed tomography; T1w, T1 weighted.

Table IV. Correlations between the SUV and ADC, and D, D* and $\mathrm{f}$ values.

\begin{tabular}{lcc}
\hline Correlation & $\mathrm{r}$ & P-value \\
\hline SUVmax and ADCmin & -0.025 & 0.892 \\
SUVmax and D & -0.107 & 0.553 \\
SUVmax and D* & -0.060 & 0.741 \\
SUVmax and f & -0.159 & 0.378 \\
SUVmean and ADCmean & 0.014 & 0.936 \\
SUVmean and D & -0.102 & 0.573 \\
SUVmean and D* & -0.057 & 0.753 \\
SUVmean and f & -0.186 & 0.300 \\
\hline
\end{tabular}

SUV, standardized uptake values; ADC, apparent diffusion coefficient; D, true diffusion coefficient; D*, pseudo-diffusion coefficient.

conspicuity was significantly higher in T2w PET/MR imaging than in PET/CT. However, the quality of PET/MR imaging was enough to delineate lesions relative to the surrounding soft tissue. Considering the potential infiltration of NPC into the adjacent structures, such as the prevertebral muscle, internal carotid artery and skull base, as well as intracranial spread, PET/MR showed more reliable determination of tumor tissue.

FDG PET/CT has a growing role in the diagnosis and management of NPC. The clinical value of ${ }^{18} \mathrm{~F}-\mathrm{FDG} \mathrm{PET} / \mathrm{CT}$ for evaluating the T stage of patients with NPC is limited by the spillover effect of ${ }^{18} \mathrm{~F}-\mathrm{FDG}$, low spatial resolution and imaging misregistration $(2,48)$. The present study showed that the tumor conspicuity and the diagnostic confidence of PET/MR were significantly higher than those of PET/CT. These parameters are of particular importance because the $\mathrm{T}$ classification of NPC depends on the infiltration of adjacent structures. A previous study also showed that ${ }^{18} \mathrm{~F}$-FDG PET/MR is more accurate than head and neck MRI and PET/CT in terms of tumor staging (39).

NPC is prone to cervical lymph node metastasis and the retropharyngeal lymph nodes are considered first echelon nodes (1). Accurate detection of lymph node metastasis is important for N staging (41) in NPC. In the present study, a total of 64 suspicious metastatic lymph nodes were detected by T2w PET/MR. However, PET/CT and T1w PET/MR imaging detected 61 lymph nodes, apart from three retropharyngeal lymph nodes. Since the three retropharyngeal lymph nodes were merging with the primary tumors, PET/CT and T1w
PET/MR imaging could not separate the retropharyngeal lymph nodes from the primary tumors, while a clear distinction between the primary tumor and the attached retropharyngeal lymph nodes was achieved using T2w PET/MR imaging.

Considering the conspicuity of the lymph nodes, the present results showed that T1w PET/MR imaging and T2w $\mathrm{PET} / \mathrm{MR}$ imaging were more efficient than PET/CT. However, a previous study reported no significant difference between contrast-enhanced PET/CT, T2w PET/MR imaging, and contrast-enhanced PET/MR in the detection and conspicuity of cervical lymph nodes (33). This discrepancy may be due to the fact that $42.1 \%(32 / 76)$ of the lymph nodes were retropharyngeal in the present study, and CT without contrast medium does not define the margins in retropharyngeal lymph nodes. Since MR has high soft tissue contrast, especially T2w MR imaging with fat suppression, it is efficient to detect and to delineate lymph nodes, and this modality is also adequate for the detection of cystic or necrotic lymph nodes.

Intensity-modulated radiotherapy (IMRT) is the primary and preferred treatment method for NPC (1), and the accurate delineation of target volumes and normal organs relies on clear lesion borders. CT, MR and MR/CT fusion imaging are the basic methods for delineating the target volumes, and $\mathrm{PET} / \mathrm{CT}$ facilitates radiation therapy planning (49). Moreover, in the present study, it was showed that T1w PET/MR imaging and T2w PET/MR imaging performed significantly better than PET/CT with respect of the conspicuity of the primary tumors and lymph nodes. PET/MR delineated more lesion borders, and it may be possible to determine and deliver the most appropriate radiation dose level to different parts of the target volume with IMRT. The volume of interest (VOI) of DWI and the VOI of FDG PET were not completely overlapping and the volume defined by cluster analysis might be useful for dose painting (40), which has been applied in radiotherapy for NPC.

Whether SUV and ADC calculated by conventional mono-exponential DWI are statistically correlated or independent biomarkers in tumors has been investigated by numerous previous studies $(8-19,40)$. Cao et al (40) reported that no significant correlation between SUV and ADC was observed in NPC. The present results were concordant with these previous findings, indicating that these two biomarkers are independent in NPC; the present results are also in line with previous studies in HNSCC $(8,17,19)$. However, Nakajo et al (18) found a significant negative correlation between SUVmax and $\mathrm{ADC}_{800}$ in 26 patients with HNSCC, while Choi et al (19) showed that the ADCratio $\left(\mathrm{ADC}_{2000} / \mathrm{ADC}_{1000}\right)$ was significantly and 
A

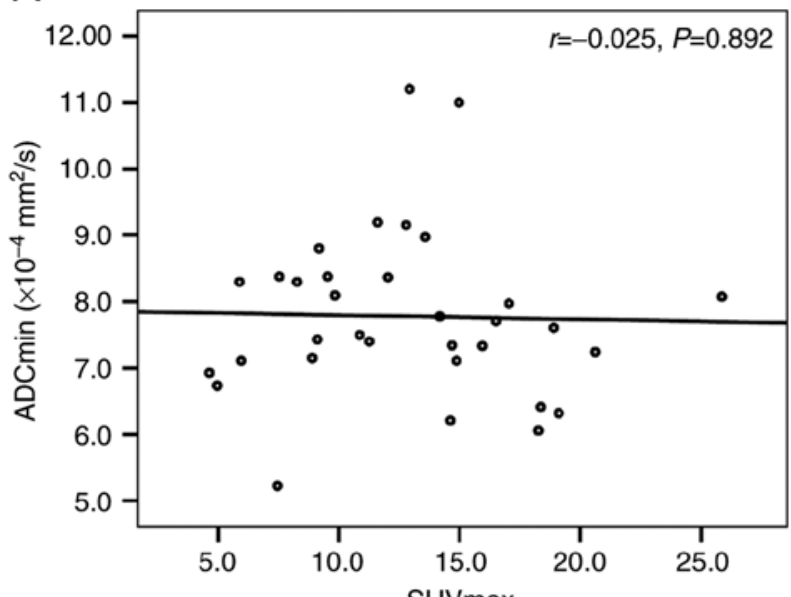

C

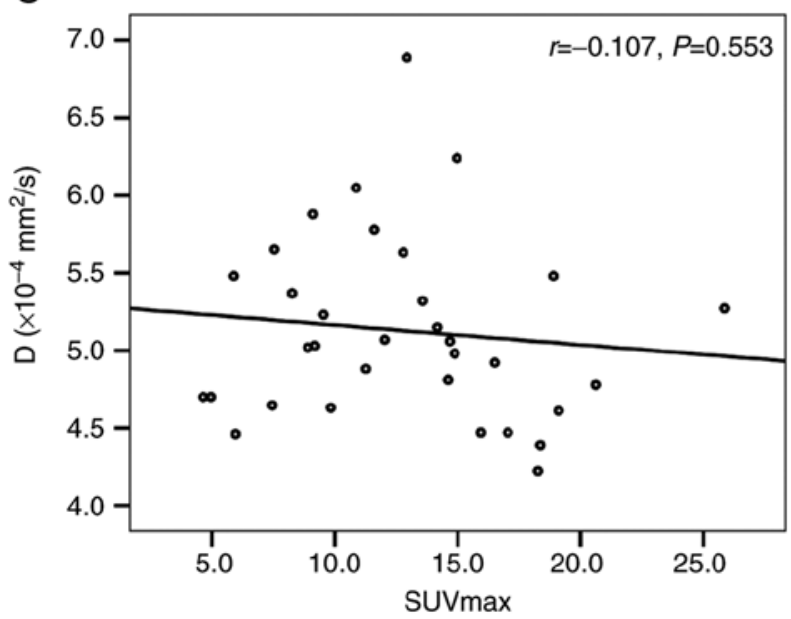

B

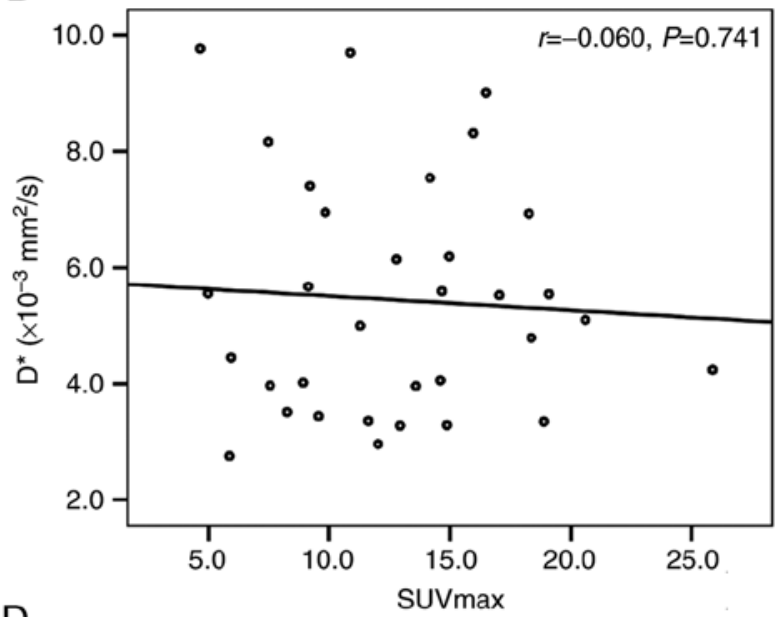

D

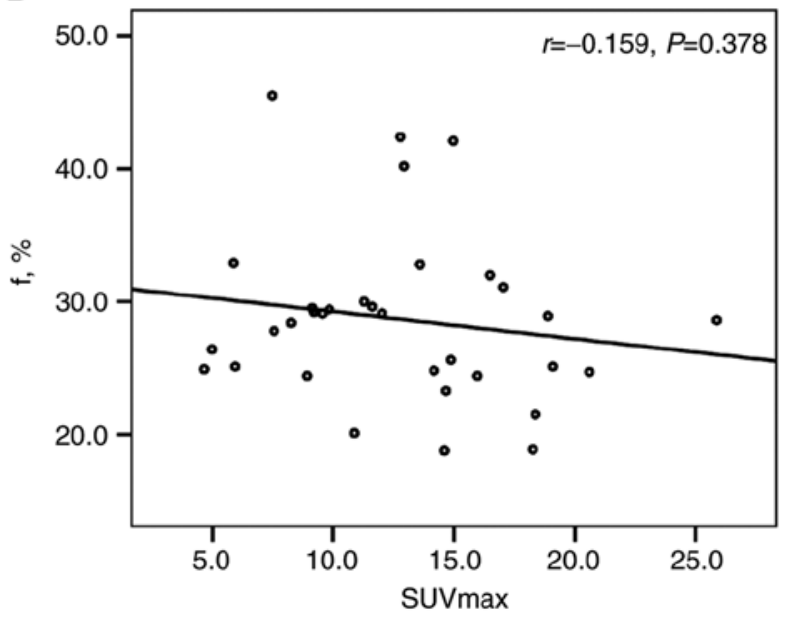

Figure 3. Correlation analysis. Scatter plots of the correlation between (A) SUVmax and ADCmin, (B) SUVmax and D*, (C) SUVmax and D, and (D) SUVmax and $\mathrm{f} \%$. No statistically significant correlation was found. SUV, standardized uptake values; ADC, apparent diffusion coefficient; D, true diffusion coefficient; $\mathrm{D}^{*}$, pseudo-diffusion coefficient.

positively correlated to SUV. There were some differences in these previous studies in the interval times between PET and MR scans, which ranged between 3 days and 2 weeks. In the present study, PET and MR scanning were performed on the same day using sequential PET/MR. Therefore, the correlation between SUV and ADC may be made more reliable by shortening the time interval between PET and MR analyses.

Traditional DWI via the conventional mono-exponential model cannot distinguish between true diffusion and microcirculatory perfusion (27). It has been confirmed that microcirculation of the blood or perfusion in capillary networks can substantially affect the measurement of ADC values (21). According to the IVIM theory, D represents true molecular diffusion, while $\mathrm{D}^{*}$ is proportional to the average blood velocity and the mean capillary segment length (50), which indicates that $\mathrm{D}^{*}$ is linked to vascularity and perfusion in tissues. The f value is another perfusion-related parameter corresponding to the fractional volume (percentage) of capillary blood flow (27). Therefore, whether IVIM parameters ( $D^{*}, \mathrm{D}$ and $\mathrm{f}$ values) had any significant correlation with SUV in NPC were investigated in the present, and IVIM showed the ability to separately quantitate the diffusion and perfusion effects.
The present preliminary results showed that there was no significant correlation between the SUV and D*, D and $f$ values. ADC represents the total diffusion value and $\mathrm{D}$ represents true diffusion, and there was no correlation between these two factors with SUV regarding cellular glucose metabolism. Furthermore, no relationship was found between cellular glucose metabolism and cellular perfusion in the present study. The SUV and D, D*, and f values are independent biomarkers in NPC. Although the possible correlation between SUV and ADC remains controversial, and there was no significant correlation between the SUV and D*, D and $\mathrm{f}$ values in NPC, these parameters are all biomarkers that may potentially predict early treatment responses. Additional work should investigate the correlation between the SUV and D*, D and $f$ values in other tumors.

Notably, the present study had several limitations. No contrast medium was applied during MRI and CT, therefore, it was not possible to analyze the lesion characterization in cePET/CT and cePET/MR imaging. In addition, the PET/MR comprised only a head and neck protocol, so no other body were analyzed by the MR imaging to evaluate distant metastasis. Moreover, the patient cohort was relatively small, and all NPC patients presented undifferentiated carcinomas. 
Table V. Lesion conspicuity in the three imaging techniques.

\begin{tabular}{|c|c|c|c|c|c|c|}
\hline \multirow[b]{2}{*}{ Grade } & \multicolumn{3}{|c|}{ Primary tumors } & \multicolumn{3}{|c|}{ Lymph nodes } \\
\hline & $\begin{array}{c}\mathrm{PET} / \mathrm{CT} \\
(\%)\end{array}$ & $\begin{array}{c}\text { T1w PET/MR } \\
(\%)\end{array}$ & $\begin{array}{c}\text { T2w PET/MR } \\
(\%)\end{array}$ & $\begin{array}{c}\mathrm{PET} / \mathrm{CT} \\
(\%)\end{array}$ & $\begin{array}{c}\text { T1w PET/MR } \\
(\%)\end{array}$ & $\begin{array}{c}\mathrm{T} 2 \mathrm{w} \text { PET/MR } \\
(\%)\end{array}$ \\
\hline Poor & 28.6 & 0 & 0 & 15.6 & 4.7 & 0 \\
\hline Moderate & 48.6 & 14.3 & 0 & 15.6 & 3.1 & 0 \\
\hline Well & 22.8 & 40.0 & 14.3 & 20.3 & 10.9 & 1.6 \\
\hline Excellent & 0 & 45.7 & 85.7 & 48.5 & 81.3 & 98.4 \\
\hline
\end{tabular}

PET, positron emission tomography; MR, magnetic resonance imaging; CT, computed tomography; T1w, T1 weighted.
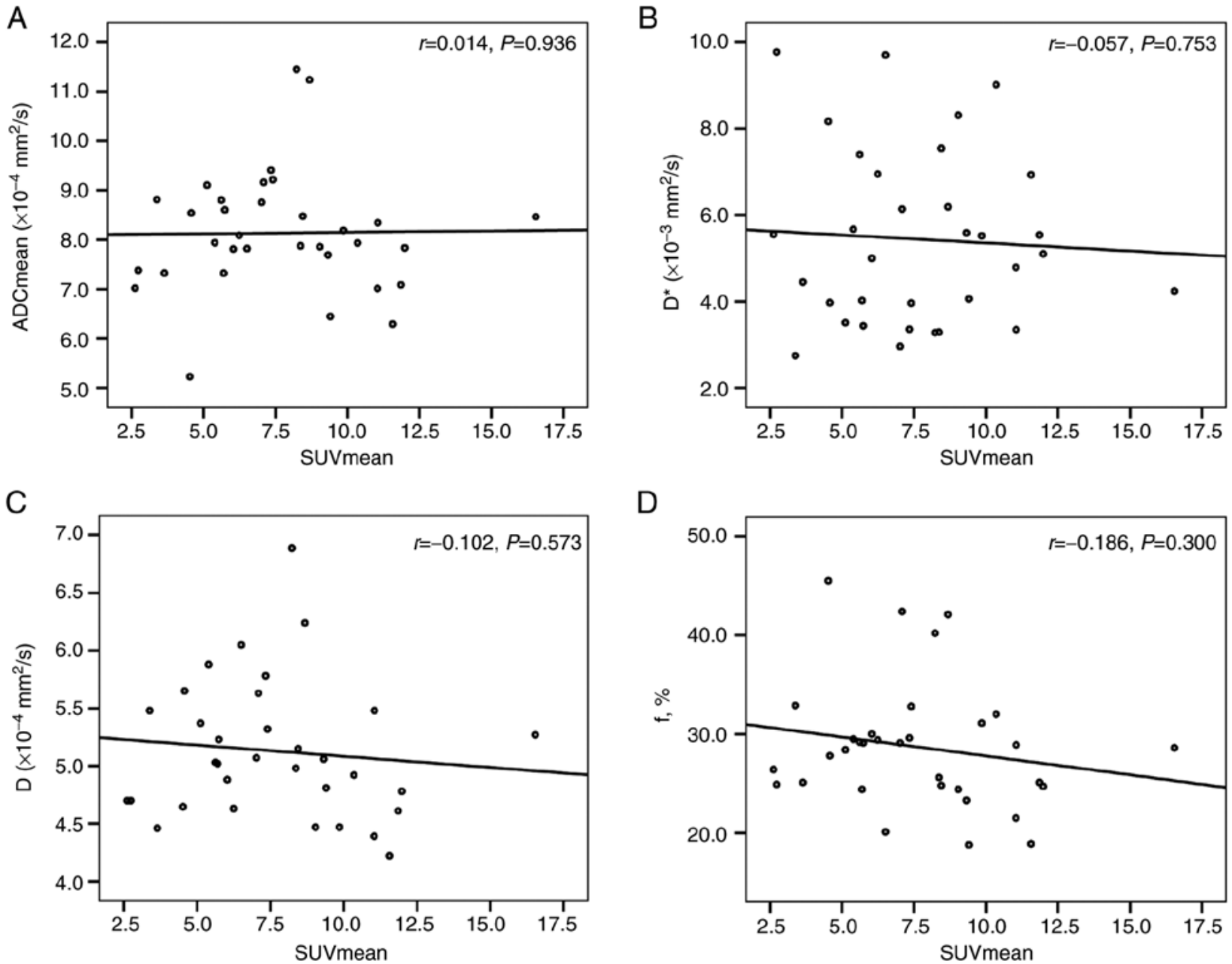

Figure 4. Correlation analysis. Scatter plots of the correlation between (A) SUVmean and ADCmean, (B) SUVmean and D*, (C) SUVmean and D, and (D) SUVmean and $\mathrm{f} \%$. No statistically significant correlation was found between the SUVmean and ADCmean, $\mathrm{D}^{*}, \mathrm{D}$ and $\mathrm{f}$ values. SUV, standardized uptake values; ADC, apparent diffusion coefficient; D, true diffusion coefficient; $\mathrm{D}^{*}$, pseudo-diffusion coefficient.

Therefore, a larger sample size should be used for further investigation and the correlation between the SUV and ADC, $\mathrm{D}, \mathrm{D}^{*}$ and $\mathrm{f}$ values of differentiated carcinomas should be further analyzed. Further studies are required to analyze whether the biomarkers of NPC may show a correlation with tumor differentiation.

The present study suggested that PET/MR was more efficient in the visualization and characterization of NPC compared with $\mathrm{PET} / \mathrm{CT}$. As a result, PET/MR showed high lesion detection and good image quality. Additionally, PET/MR offered higher lesion conspicuity and diagnostic confidence for NPC, which may facilitate radiotherapy planning and tumor classification. However, no correlations were identified between the SUV assessed by ${ }^{18} \mathrm{~F}$-FDG PET/CT, and the ADC, D, D* and $f$ values determined by IVIM with multiple b values in NPC. The present findings suggested that SUV and ADC, D, D* and f values are independent biomarkers in NPC, and they may provide different information, thus facilitating a correct diagnosis. 
A

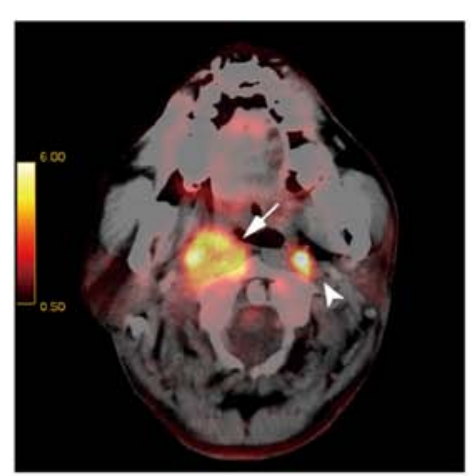

D

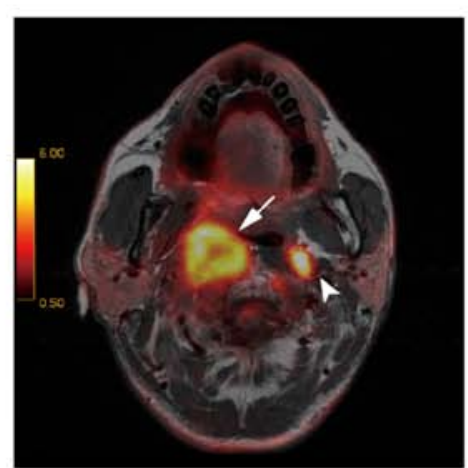

B

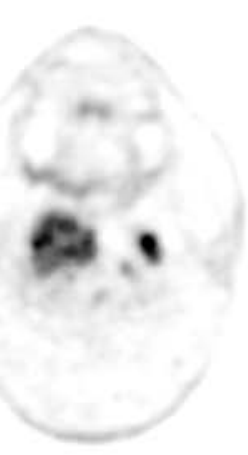

E

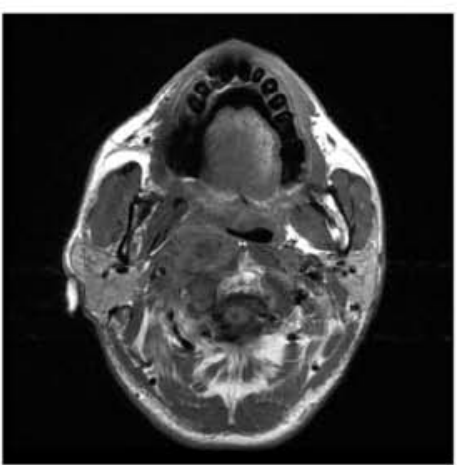

C

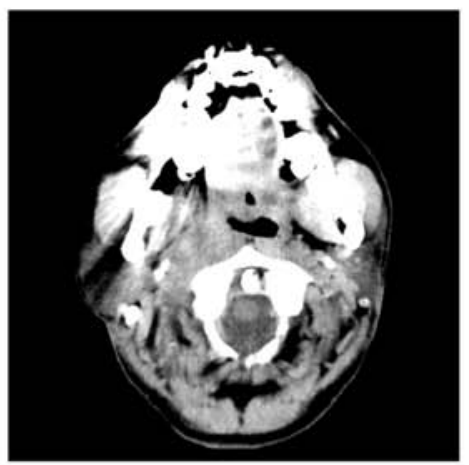

F

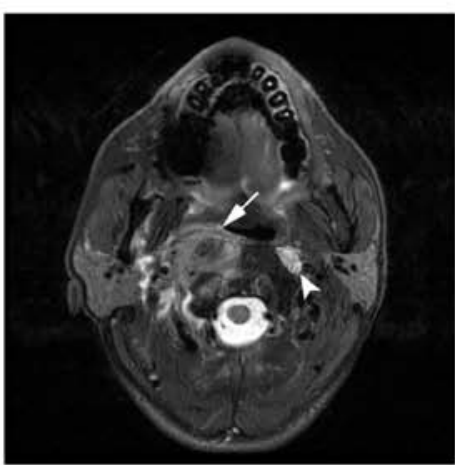

Figure 5. A 38-year-old male patient suffering from prevertebral muscle invasion of NPC. Prevertebral muscle invasion of NPC is indicated by an arrow. (A) Image artefacts caused by dental implants that affected the PET/CT. (B) PET imaging. (C) CT scan. (D) PET/MR and (E) MR were not affected by artefacts. (F) T2w imaging could delineate the margin of the retropharyngeal lymph node, indicated by an arrowhead. PET, positron emission tomography; MR, magnetic resonance imaging; CT, computed tomography; T1w, T1 weighted.

A

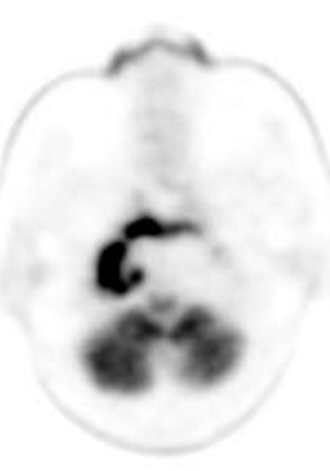

D

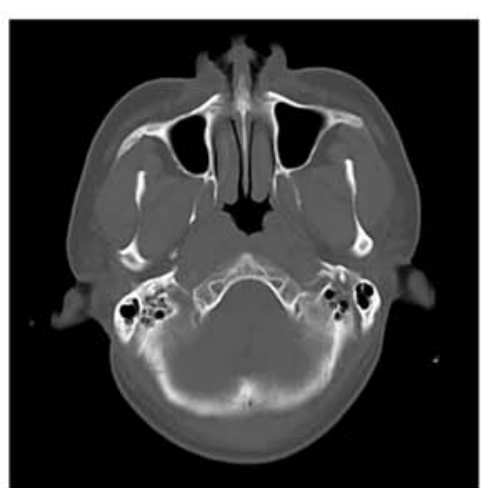

$\mathrm{B}$

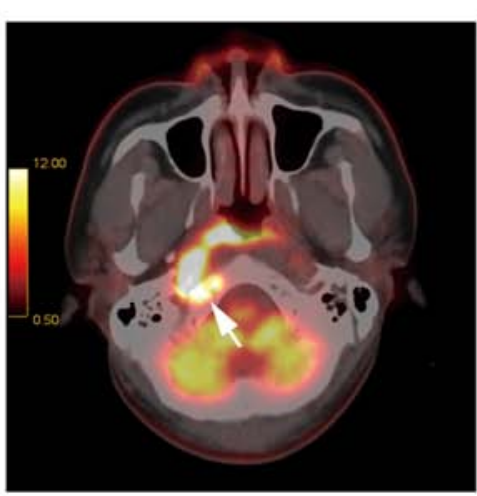

E

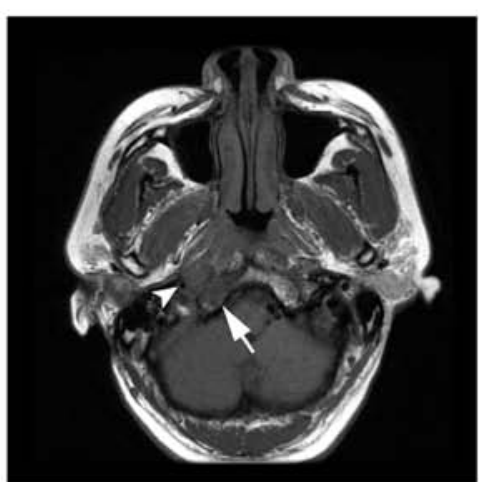

C

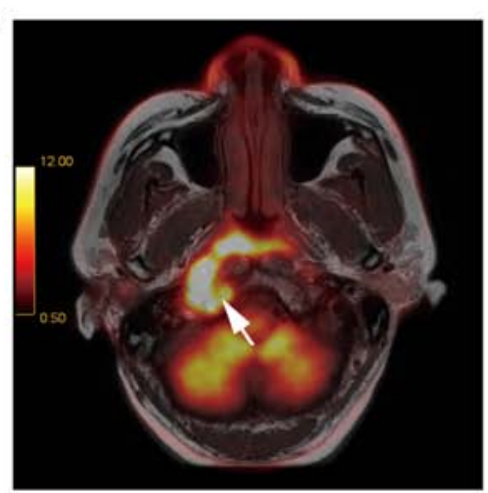

$\mathrm{F}$

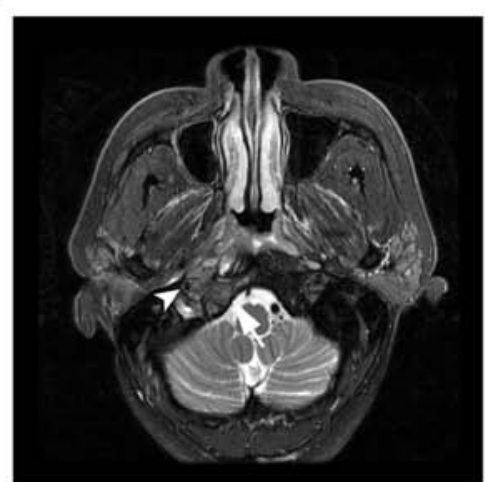

Figure 6. Infiltration of the adjacent structures by PET/CT and PET/MR in a 39-year-old male patient. (A) PET, (B) PET/CT and (C) T1w PET/MR imaging showed the high uptake of the lesions. However, (D) CT showed no destruction of the occipital bone. (E) MR revealed marrow infiltration (indicated by an arrow) with hypointense areas on (F) T1w imaging and hyperintense areas on fat suppression T2w imaging. MR showed that the right internal carotid artery was surrounded by lesions (indicated by an arrowhead). PET, positron emission tomography; MR, magnetic resonance imaging; CT, computed tomography; T1w, T1 weighted. 


\section{Acknowledgements}

The authors would like to thank Professor Hansheng Lin (Department of Medical Statistics, School of Medicine, Jinan University) for his excellent statistics support.

\section{Funding}

The present study was financially supported by the National Natural Science Foundation of China (grant no. 81871383), the Medical Scientific Research Foundation of Guangdong Province (grant no. A2018135), the Fundamental Research Funds for the Central Universities (grant no. 21617311) and the Science and Technology Program of Guangzhou (grant no. 201804010440).

\section{Availability of data and materials}

Additional data are available from the corresponding author upon reasonable request.

\section{Authors' contributions}

YC, LB and HX conceived the present study. YC, LB, JS and YT collected and analyzed the data. YC, LB, JS, YT, XL, BG, JG and LW analyzed the data. YC and LB drafted the manuscript. All authors read and approved the final manuscript.

\section{Ethics approval and consent to participate}

The present study was approved by The Ethics Committee of The First Affiliated Hospital of Jinan University.

\section{Patient consent for publication}

Not applicable.

\section{Competing interests}

The authors declare that they have no competing interests.

\section{References}

1. Chua MLK, Wee JTS, Hui EP and Chan ATC: Nasopharyngeal carcinoma. Lancet 387: 1012-1024, 2016

2. Lee AW, Ma BB, Ng WT and Chan AT: Management of nasopharyngeal carcinoma: Current practice and future perspective. J Clin Oncol 33: 3356-3364, 2015.

3. Chang KP, Tsang NM, Liao CT, Hsu CL, Chung MJ, Lo CW, Chan SC, Ng SH, Wang HM and Yen TC: Prognostic significance of ${ }^{18} \mathrm{~F}$-FDG PET parameters and plasma Epstein-Barr virus DNA load in patients with nasopharyngeal carcinoma. J Nucl Med 53: 21-28, 2012.

4. Yang Z, Shi Q, Zhang Y, Pan H, Yao Z, Hu S, Shi W, Zhu B, Zhang Y and Hu C: Pretreatment ${ }^{18}$ F-FDG uptake heterogeneity can predict survival in patients with locally advanced nasopharyngeal carcinoma-a retrospective study. Radiat Oncol 10: 4, 2015.

5. Vellayappan BA,Soon YY,EarnestA,ZhangQ,KohWY,ThamIW and Lee KM: Accuracy of ${ }^{18} \mathrm{~F}$-flurodeoxyglucose-positron emission tomography/computed tomography in the staging of newly diagnosed nasopharyngeal carcinoma: A systematic review and meta-analysis. Radiol Oncol 48: 331-338, 2014.

6. Chua ML, Ong SC, Wee JT, Ng DC, Gao F, Tan TW, Fong KW, Chua ET, Khoo JB and Low JS: Comparison of 4 modalities for distant metastasis staging in endemic nasopharyngeal carcinoma. Head Neck 31: 346-354, 2009.
7. Chang MC, Chen JH, Liang JA, Yang KT, Cheng KY and Kao CH: Accuracy of whole-body FDG-PET and FDG-PET/CT in $\mathrm{M}$ staging of nasopharyngeal carcinoma: A systematic review and meta-analysis. Eur J Radiol 82: 366-373, 2013.

8. Varoquaux A, Rager O, Lovblad KO, Masterson K, Dulguerov $P$, Ratib O, Becker CD and Becker M: Functional imaging of head and neck squamous cell carcinoma with diffusion-weighted MRI and FDG PET/CT: Quantitative analysis of ADC and SUV. Eur J Nucl Med Mol Imaging 40: 842-852, 2013.

9. Regier M, Derlin T, Schwarz D, Laqmani A, Henes FO, Groth M, Buhk JH, Kooijman H and Adam G: Diffusion weighted MRI and ${ }^{18} \mathrm{~F}$-FDG PET/CT in non-small cell lung cancer (NSCLC): Does the apparent diffusion coefficient (ADC) correlate with tracer uptake (SUV). Eur J Radiol 81: 2913-2918, 2012.

10. Baba S, Isoda T, Maruoka Y, Kitamura Y, Sasaki M, Yoshida T and Honda $\mathrm{H}$ : Diagnostic and prognostic value of pretreatment SUV in ${ }^{18} \mathrm{~F}-\mathrm{FDG} / \mathrm{PET}$ in breast cancer: Comparison with apparent diffusion coefficient from diffusion-weighted MR imaging. J Nucl Med 55: 736-742, 2014.

11. Nakajo M, Kajiya Y, Kaneko T, Kaneko Y, Takasaki T, Tani A, Ueno M, Koriyama $C$ and Nakajo M: FDG PET/CT and diffusion-weighted imaging for breast cancer: Prognostic value of maximum standardized uptake values and apparent diffusion coefficient values of the primary lesion. Eur J Nucl Med Mol Imagin 37: 2011-2020, 2010.

12. Kitajima K, Yamano T, Fukushima K, Miyoshi Y, Hirota S, Kawanaka Y, Miya M, Doi H, Yamakado K and Hirota S: Correlation of the SUVmax of FDG-PET and ADC values of diffusion-weighted MR imaging with pathologic prognostic factors in breast carcinoma. Eur J Radiol 85: 943-949, 2016.

13. Wong CS, Gong N, Chu YC, Anthony MP, Chan Q, Lee HF, Chu KM and Khong PL: Correlation of measurements from diffusion weighted MR imaging and FDG PET/CT in GIST patients: ADC versus SUV. Eur J Radiol 81: 2122-2126, 2012.

14. Brandmaier P, Purz S, Bremicker K, Höckel M, Barthel H, Kluge R, Kahn T, Sabri O and Stumpp P: Simultaneous $\left[{ }^{18} \mathrm{~F}\right]$ FDG-PET/MRI: Correlation of apparent diffusion coefficient (ADC) and standardized uptake value (SUV) in primary and recurrent cervical cancer. PLoS One 10: e0141684, 2015.

15. Wu X, Korkola P, Pertovaara H, Eskola H, Järvenpää R and Kellokumpu-Lehtinen PL: No correlation between glucose metabolism and apparent diffusion coefficient in diffuse large B-cell lymphoma: A PET/CT and DW-MRI study. Eur J Radiol 79: e117-e121, 2011.

16. Giraudo C, Karanikas G, Weber M, Raderer M, Jaeger U, Simonitsch-Klupp I and Mayerhoefer ME: Correlation between glycolytic activity on [18F]-FDG-PET and cell density on diffusion-weighted MRI in lymphoma at staging. J Magn Reson Imaging 47: 1217-1226, 2018

17. Fruehwald-Pallamar J, Czerny C, Mayerhoefer ME, Halpern BS, Eder-Czembirek C, Brunner M, Schuetz M, Weber M, Fruehwald L and Herneth AM: Functional imaging in head and neck squamous cell carcinoma: Correlation of PET/CT and diffusion-weighted imaging at 3 tesla. Eur J Nucl Med Mol Imaging 38: 1009-1019, 2011.

18. Nakajo M, Nakajo M, Kajiya Y, Tani A, Kamiyama T, Yonekura R, Fukukura Y, Matsuzaki T, Nishimoto $K$, Nomoto $M$ and Koriyama C: FDG PET/CT and diffusion-weighted imaging of head and neck squamous cell carcinoma: Comparison of prognostic significance between primary tumor standardized uptake value and apparent diffusion coefficient. Clin Nucl Med 37: 475-480, 2012.

19. Choi SH, Paeng JC, Sohn CH, Pagsisihan JR, Kim YJ, Kim KG, Jang JY, Yun TJ, Kim JH, Han MH and Chang KH: Correlation of ${ }^{18}$ F-FDG uptake with apparent diffusion coefficient ratio measured on standard and high b value diffusion MRI in head and neck cancer. J Nucl Med 52: 1056-1062, 2011.

20. Dixon WT: Separation of diffusion and perfusion in intravoxel incoherent motion MR imaging: A modest proposal with tremendous potential. Radiology 168: 566-567, 1988.

21. Le Bihan D, Breton E, Lallemand D, Aubin ML, Vignaud J and Laval-Jeantet M: Separation of diffusion and perfusion in intravoxel incoherent motion MR imaging. Radiology 168: 497-505, 1988.

22. Le Bihan D, Breton E, Lallemand D, Grenier P, Cabanis E and Laval-Jeantet M: MR imaging of intravoxel incoherent motions: Application to diffusion and perfusion in neurologic disorders. Radiology 161: 401-407, 1986.

23. Liu C, Liang C, Liu Z, Zhang S and Huang B: Intravoxel incoherent motion (IVIM) in evaluation of breast lesions: Comparison with conventional DWI. Eur J Radiol 82: e782-e789, 2013. 
24. Zhang SX, Jia QJ, Zhang ZP, Liang $\mathrm{CH}$, Chen WB, Qiu QH and Li H: Intravoxel incoherent motion MRI: Emerging applications for nasopharyngeal carcinoma at the primary site. Eur Radiol 24 1998-2004, 2014.

25. Jia QJ, Zhang SX, Chen WB, Liang L, Zhou ZG, Qiu QH, Liu ZY, Zeng QX and Liang CH: Initial experience of correlating parameters of intravoxel incoherent motion and dynamic contrast-enhanced magnetic resonance imaging at $3.0 \mathrm{~T}$ in nasopharyngeal carcinoma. Eur Radiol 24: 3076-3087, 2014

26. Lai V, Li X, Lee VH, Lam KO, Fong DY, Huang B, Chan Q and Khong PL: Nasopharyngeal carcinoma: Comparison of diffusion and perfusion characteristics between different tumour stages using intravoxel incoherent motion MR imaging. Eur Radiol 24 176-183, 2014.

27. Yu XP, Hou J, Li FP, Wang H, Hu PS, Bi F and Wang W: Intravoxel incoherent motion diffusion weighted magnetic resonance imaging for differentiation between nasopharyngeal carcinoma and lymphoma at the primary site. J Comput Assist Tomogr 40: 413-418, 2016.

28. Xiao-Ping Y, Jing H, Fei-ping L, Yin H, Qiang L, Lanlan W and Wei W: Intravoxel incoherent motion MRI for predicting early response to induction chemotherapy and chemoradiotherapy in patients with nasopharyngeal carcinoma. J Magn Reson Imaging 43: 1179-1190, 2016

29. Xiao Y, Pan J, Chen Y, Chen Y, He Z and Zheng X: Intravoxel incoherent motion-magnetic resonance imaging as an early predictor of treatment response to neoadjuvant chemotherapy in locoregionally advanced nasopharyngeal carcinoma. Medicine (Baltimore) 94: e973, 2015.

30. Queiroz MA and Huellner MW: PET/MR in cancers of the head and neck. Semin Nucl Med 45: 248-265, 2015

31. Spick C, Herrmann K and Czernin J: ${ }^{18}$ F-FDG PET/CT and PET/MRI perform equally well in cancer: Evidence from studies on more than 2,300 patients. J Nucl Med 57: 420-430, 2016.

32. Queiroz MA, Hüllner M, Kuhn F, Huber G, Meerwein C, Kollias S, von Schulthess G and Veit-Haibach P: PET/MRI and PET/CT in follow-up of head and neck cancer patients. Eur J Nucl Med Mol Imaging 41: 1066-1075, 2014.

33. Kuhn FP, Hüllner M, Mader CE, Kastrinidis N, Huber GF, von Schulthess GK, Kollias $S$ and Veit-Haibach $P$. Contrast-enhanced PET/MR imaging versus contrast-enhanced $\mathrm{PET} / \mathrm{CT}$ in head and neck cancer: How much MR information is needed. J Nucl Med 55: 551-558, 2014.

34. Kubiessa K, Purz S, Gawlitza M, Kühn A, Fuchs J, Steinhoff KG, Boehm A, Sabri O, Kluge R, Kahn T and Stumpp P: Initial clinical results of simultaneous ${ }^{18} \mathrm{~F}$-FDG PET/MRI in comparison to ${ }^{18}$ F-FDG PET/CT in patients with head and neck cancer. Eur J Nucl Med Mol Imaging 41: 639-648, 2014.

35. Partovi S, Kohan A, Vercher-Conejero JL, Rubbert C, Margevicius S, Schluchter MD, Gaeta C, Faulhaber P and Robbin MR: Qualitative and quantitative performance of ${ }^{18} \mathrm{~F}-\mathrm{FDG}-\mathrm{PET} / \mathrm{MRI}$ versus ${ }^{18} \mathrm{~F}-\mathrm{FDG}-\mathrm{PET} / \mathrm{CT}$ in patients with head and neck cancer. AJNR Am J Neuroradiol 35: 1970-1975, 2014.

36. Varoquaux A, Rager O, Poncet A, Delattre BM, Ratib O, Becker CD, Dulguerov P, Dulguerov N, Zaidi H and Becker M: Detection and quantification of focal uptake in head and neck tumours: ${ }^{18}$ F-FDG PET/MR versus PET/CT. Eur J Nucl Med Mol Imaging 41: 462-475, 2014

37. Covello M, Cavaliere C, Aiello M, Cianelli MS, Mesolella M, Iorio B, Rossi A and Nicolai E: Simultaneous PET/MR head-neck cancer imaging: Preliminary clinical experience and multiparametric evaluation. Eur J Radiol 84: 1269-1276, 2015.
38. Schaarschmidt BM, Heusch P, Buchbender C, Ruhlmann M, Bergmann C, Ruhlmann V, Schlamann M, Antoch G, Forsting M and Wetter A: Locoregional tumour evaluation of squamous cell carcinoma in the head and neck area: A comparison between MRI, PET/CT and integrated PET/MRI. Eur J Nucl Med Mol Imaging 43: 92-102, 2016.

39. Chan SC, Yeh CH, Yen TC, Ng SH, Chang JT, Lin CY, Yen-Ming T, Fan KH, Huang BS, Hsu CL, et al: Clinical utility of simultaneous whole-body 18F-FDG PET/MRI as a single-step imaging modality in the staging of primary nasopharyngeal carcinoma. Eur J Nucl Med Mol Imaging 45: 1297-1308, 2018

40. Cao C, Yang P, Xu Y, Niu T, Hu Q and Chen X: Feasibility of multiparametric imaging with PET/MR in nasopharyngeal carcinoma: A pilot study. Oral Oncol 93: 91-95, 2019.

41. Edge SB, Byrd DR, Compton CC, Fritz AG, Greene F, Trotti A (eds): AJCC Cancer Staging Manual. 7th edition. Springer, New York, NY, 2010.

42. Prakash P, Kalra MK, Digumarthy SR, Hsieh J, Pien H, Singh S, Gilman MD and Shepard JA: Radiation dose reduction with chest computed tomography using adaptive statistical iterative reconstruction technique: Initial experience. J Comput Assist Tomogr 34: 40-45, 2010.

43. Rodrigues RS, Bozza FA, Christian PE, Hoffman JM, Butterfield RI, Christensen CR, Heilbrun M, Wiggins RH 3rd, Hunt JP, Bentz BG, et al: Comparison of whole-body PET/CT, dedicated high-resolution head and neck PET/CT, and contrast-enhanced $\mathrm{CT}$ in preoperative staging of clinically M0 squamous cell carcinoma of the head and neck. J Nucl Med 50: 1205-1213, 2009.

44. Abdel Khalek Abdel Razek A and King A: MRI and CT of nasopharyngeal carcinoma. AJR Am J Roentgenol 198: 11-18, 2012.

45. Le Bihan D, Turner R and MacFall JR: Effects of intravoxel incoherent motions (IVIM) in steady-state free precession (SSFP) imaging: Application to molecular diffusion imaging. Magn Reson Med 10: 324-337, 1989.

46. King AD, Vlantis AC, Bhatia KS, Zee BC, Woo JK, Tse GM, Chan AT and Ahuja AT: Primary nasopharyngeal carcinoma: Diagnostic accuracy of MR imaging versus that of endoscopy and endoscopic biopsy. Radiology 258: 531-537, 2011.

47. Chen YK, Su CT, Ding HJ, Chi KH, Liang JA, Shen YY, Chen LK, Yeh CL, Liao AC and Kao CH: Clinical usefulness of fused PET/CT compared with PET alone or CT alone in nasopharyngeal carcinoma patients. Anticancer Res 26: 1471-1477, 2006.

48. Ng SH, Chan SC, Yen TC, Chang JT, Liao CT, Ko SF, Liu FY, Chin SC, Fan KH and Hsu CL: Staging of untreated nasopharyngeal carcinoma with PET/CT: Comparison with conventional imaging work-up. Eur J Nucl Med Mol Imaging 36: 12-22, 2009.

49. Mohandas A, Marcus C, Kang H, Truong MT and Subramaniam RM: FDG PET/CT in the management of nasopharyngeal carcinoma. AJR Am J Roentgenol 203: W146-W157, 2014

50. Le Bihan D and Turner R: The capillary network: A link between IVIM and classical perfusion. Magn Reson Med 27: 171-178, 1992.

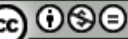

This work is licensed under a Creative Commons Attribution-NonCommercial-NoDerivatives 4.0 International (CC BY-NC-ND 4.0) License. 